A N N A L E S Annales de Bretagne et des Pays de l'Ouest

Anjou. Maine. Poitou-Charente. Touraine

$119-1 \mid 2012$

Varia

\title{
Les Pérégrinations de Saint-Philibert
}

\section{Sébastien Legros}

\section{OpenEdition}

Journals

Édition électronique

URL : http://journals.openedition.org/abpo/2334

DOI : $10.4000 /$ abpo.2334

ISBN : 978-2-7535-1852-0

ISSN : 2108-6443

Éditeur

Presses universitaires de Rennes

Édition imprimée

Date de publication : 30 mars 2012

Pagination : 192-194

ISBN : 978-2-7535-1850-6

ISSN : 0399-0826

Référence électronique

Sébastien Legros, "Les Pérégrinations de Saint-Philibert», Annales de Bretagne et des Pays de l'Ouest [En ligne], 119-1 | 2012, mis en ligne le, consulté le 23 septembre 2020. URL : http:// journals.openedition.org/abpo/2334 ; DOI : https://doi.org/10.4000/abpo.2334

Ce document a été généré automatiquement le 23 septembre 2020.

(c) Presses universitaires de Rennes 


\title{
Les Pérégrinations de Saint-Philibert
}

\author{
Sébastien Legros
}

\section{RÉFÉRENCE}

Isabelle Cartron, Les Pérégrinations de Saint-Philibert. Genèse d'un réseau monastique dans la société carolingienne, Rennes, PUR, 2009, 456 p. (ISBN 978-2-7535-0955-9).

1 Cet ouvrage est l'édition d'une thèse de doctorat soutenue en 1998 à l'université d'Aixen-Provence. L'auteure associe les perspectives historiques et archéologiques afin de traiter son sujet: la genèse d'un réseau monastique dans la société carolingienne. Ce réseau monastique est celui de l'abbaye Saint-Philibert de Tournus, dont l'auteure vient utilement reconstruire l'origine et l'organisation, en contrepoint au modèle souvent perçu comme fondateur de l'Ecclesia cluniacensis. C'est là le grand mérite de cette thèse, que de montrer que le haut Moyen Âge connaissait, avant même la structure rigide de l'ordre clunisien, l'organisation souple mais déjà hiérarchisée du réseau monastique.

2 La première partie étudie le parcours des moines à partir de l'île de Noirmoutier (Herio) : sous la menace normande, les moines - et leurs reliques - passent sur le continent en 836, à Deas, puis, en 858 , au monasteriolum de Cunault, concédé par le comte de Tours, Vivien, et confirmé par Charles le Chauve en 845 ; en 862, ils se replient en Poitou, à Messais, lieu qui leur avait été concédé par le roi en 854. L'auteure opère un bilan de ces premiers déplacements, dont l'originalité réside dans le fait que les moines n'ont pas rejoint leur monastère d'origine : c'est bien la présence des reliques qui protège d'emblée la cohésion de la congrégation, tandis que sa survie matérielle est toujours garantie - anticipée? - par une aide extérieure de haut niveau. Ces déplacements ne résultent pas d'une fuite dans l'urgence, mais s'inscrivent dans un schéma politique. Herio est sous la protection du roi carolingien et constitue un instrument comme un autre entre les mains du gouvernement royal. D'où les liens très forts qui rapprochent les moines et les agents du roi dans une région marquée par la menace normande et par celle, non moins déterminante, des Bretons. L'auteure propose ici une confrontation très convaincante entre les déplacements des moines et 
le découpage de la marche de Bretagne contrôlée par les Carolingiens : elle peut ainsi réévaluer le rôle des Bretons dans les choix opérés par les moines, et éclairer les liens qui rapprochent, d'un côté les moines de Saint-Philibert et, d'un autre côté, les comtes d'Herbauge, le comte de Poitiers, ou les Rorgonides manceaux, c'est-à-dire un groupe aristocratique marqué par sa proximité avec le roi et dont l'abbé Geilon est un représentant typique. L'auteure achève son propos par une analyse éclairante de l'éventail liturgique (flabellum) de Saint-Philibert, dont la composition illustre l'association des moines, du roi et de ce groupe aristocratique.

3 Une deuxième partie est consacrée à l'hypothèse du passage de la communauté par l'Auvergne. Si les moines y possèdent des domaines importants comme Goudet et SaintPourçain, l'auteure reste circonspecte quant à l'idée d'un refuge auvergnat, dont la mention a vraisemblablement été reconstituée après la fixation à Tournus. Elle conduit de façon convaincante l'analyse diplomatique du dossier auvergnat et conclut que les nombreux faux qui le composent ont sans doute été forgés par l'abbé Geilon et l'évêque d'Autun Adalgarius à la fin du Ix $x^{\mathrm{e}}$ siècle.

4 La troisième partie s'arrête sur le retour à la stabilité à Tournus, à partir de 875 : Charles le Chauve concède alors aux moines l'abbatia de Saint-Valérien, la villa et le castrum de Tournus. La concession est solennelle et sans doute la fixation est-elle d'emblée envisagée. Là encore, l'auteure restitue brillamment l'arrière-plan politique de cette décision : l'installation est à situer dans le contexte de l'alliance entre Charles le Chauve et Boson pour le contrôle de la Lotharingie et l'auteure étudie finement les liens qui rattachent les moines (le rôle déterminant de l'abbé est à nouveau justement relevé) à Boson et son entourage. Cette installation est une réussite, notamment parce que les moines ont su préserver la dévotion locale à saint Valérien.

5 La partie suivante fait le bilan d'un demi-siècle de pérégrination. Si celle-ci a évidemment constitué une source de tension (rupture des offices et de la prière continuelle), elle a aussi été valorisée en tant qu'exode perçu comme une pénitence et offrant la possibilité de fonder de nouveaux établissements. Ainsi la réécriture de la Vita sancti Filiberti, après l'installation à Deas, valorise-t-elle la figure du saint comme fondateur de monastères. L'auteure analyse ensuite l'organisation du réseau monastique. Elle touche ici le cœur de son sujet et montre que l'idée de réseau est tangible dès la fixation à Tournus : la donation du roi fait de l'abbaye « le chef de tous les lieux qui lui ont été apportés » (p. 204). Elle chapeaute ainsi les dépendances, dont la dénomination est variable - villa, cella, monasteriolum - et où la présence d'un abbé ou d'un praepositus n'est jamais mentionnée. Les moines de ces dépendances semblent bien être des frères de Saint-Philibert, soumis à l'abbé de Tournus, mais gérant de façon autonome leurs biens. La dénomination de ces moines est délicate à cerner. L'auteure réévalue avec finesse le sens - ambigu - du terme congregatio, qui évolue avec la pérégrination des moines : d'abord classiquement attaché à un lieu, il s'associe ensuite progressivement au saint (la congregatio des moines de Philibert).

6 La quatrième et la cinquième partie observent le devenir de la communauté après la fixation à Tournus. L'expansion du réseau dans la vallée du Rhône est alors certainement encouragée par le souverain carolingien et recoupe l'alliance opérée entre Charles le Chauve et Boson. Le fait décisif réside ici dans l'attachement du monastère à Boson, y compris après son sacre, en 879. L'auteure met ainsi en évidence la corrélation spatiale existant entre les biens fonciers de la vallée du Rhône accordés par le roi Boson aux trois établissements qu'il favorise, Saint-Philibert de 
Tournus - autour de Mantaille et Albon, et de Viviers - mais aussi Saint-Oyend et SaintMartin d'Autun. Cet attachement se heurte finalement à l'échec de Boson et l'auteure mesure combien "l'épisode bosonide" (p.245) a isolé le monastère au moment où Cluny prenait son essor. Cette évolution conditionne la tonalité de la dernière partie, qui évalue - rapidement - l'adaptation du monastère aux nouveautés du XI siècle. L'auteure décrit un monastère conservateur qui perpétue son positionnement classique en faveur du pouvoir royal et développe ses liens avec les comtes de Mâcon, soutiens des derniers Carolingiens, puis avec les comtes de Chalon, qui prennent le parti des Capétiens. Le rayonnement du monastère réside dans ses reliques et Philibert est le saint qui fonde l'identité du réseau monastique. Ses reliques président à la mise en œuvre du programme de reconstruction du monastère au $\mathrm{XI}^{\mathrm{e}}$ siècle, dont l'auteure livre une synthèse archéologique : la crypte et le chevet ne remontent pas au-delà du XI siècle; c'est seulement cette partie de l'abbatiale qui aurait été consacrée en 1019. Le plan de l'édifice était toutefois sans doute déjà fixé, même si la construction de la nef s'étale tout au long du siècle. L'auteure complète son propos en observant le devenir des dépendances, notamment Deas et son programme architectural : le réseau reste dans les limites atteintes à la fin du $\mathrm{IX}^{\mathrm{e}}$ siècle et s'il est bien perçu comme un ensemble cohérent (les moines des dépendances sont considérés comme des frères de SaintPhilibert, faisant profession à Tournus), il ne constitue pas encore une entité juridique aboutie au début du XII ${ }^{e}$ siècle. L'auteure y voit la marque de la forte centralisation opérée par le monastère-chef.

7 Le grand mérite de cet ouvrage, au-delà bien sûr de la reconstitution érudite de l'histoire de la communauté de Saint-Philibert, réside dans cette analyse d'un réseau proprement carolingien. Construite au gré des déplacements des moines, cette identité carolingienne façonne le devenir de la congrégation : ses liens constamment entretenus avec le pouvoir royal, la structure de son patrimoine, souvent constitué à partir de propriétés provenant du fisc royal - par exemple autour de Cunault -, son implication dans les stratégies de contrôle territorial, notamment dans les espaces frontaliers (il faut souligner le remarquable travail de géographie historique réalisé par l'auteure). On regrettera peut-être à cet égard que cette identité, pourtant bien perçue comme une faiblesse dans le devenir de l'établissement, ne donne pas lieu à une analyse plus détaillée des difficultés du réseau de Saint-Philibert au XI siècle. On notera à cet égard l'absence symptomatique de la terminologie prieurale pour désigner les dépendances, hormis celle de Cunault (ce que l'auteure ne relève qu'incidemment p. 364, n. 102) : on en vient à penser que c'est justement le conservatisme carolingien de la congrégation qui a compliqué la formalisation juridique du réseau évoquée plus haut. Au final, la démarche synthétique de la cinquième partie, assumée par l'auteure, laisse quelque peu la lecture en suspens. On aimerait en savoir davantage sur cette "seigneurie monastique » (p. 297, 361) ponctuellement évoquée sans jamais être décrite précisément (la question de "l'encellulement seigneurial» est rapidement évacuée p. 294, n. 5) ; on pourrait formuler la même remarque au sujet du positionnement grégorien de l'abbaye (la notion de libertas, relevée p. 297 n'est pas contextualisée et aurait mérité une référence aux travaux de Jean-Hervé Foulon). En somme, c'est la question de la transition seigneuriale et grégorienne qui reste à l'arrière-plan, question importante qui, si elle n'était certes pas au cœur du sujet de l'auteure, eût malgré tout permis d'interroger l'attitude d'un monastère carolingien et l'organisation de son 
réseau face aux changements de son temps. La proximité clunisienne et de son modèle de développement offrait ici une comparaison évidente.

8 Hormis ces quelques remarques, l'ouvrage d'I. Cartron a le grand mérite de réévaluer la notion de réseau monastique et d'en remonter l'origine bien en-deçà des limites chronologiques habituellement retenues. Il illustre, avec le livre de Noëlle Deflou-Lecat, Saint-Germain d'Auxerre et ses dépendances ( $\mathrm{V}^{e}-\mathrm{VIII}{ }^{e}$ siècle) (Saint-Etienne, Publications de l'université de Saint-Etienne, 2010), la vitalité des études de la vie monastique avant l'an mil. Il offre aussi une synthèse érudite de l'histoire de la congrégation de SaintPhilibert, qui vient brillamment compléter la thèse de Benjamin Saint-Jean Vitus, Tournus, le castrum, l'abbaye, la ville, XI ${ }^{e}$-XIV ${ }^{e}$ siècle et prémices (université de Lyon 2, 2006). 